(C) 2017 IEEE. Personal use of this material is permitted. Permission from IEEE must be obtained for all other uses, in any current or future media, including reprinting/republishing this material for advertising or promotional purposes, creating new collective works, for resale or redistribution to servers or lists, or reuse of any copyrighted component of this work in other works. 


\title{
Generalized Continuous Wave Synthetic Aperture Radar for High Resolution and Wide Swath Remote Sensing
}

\author{
Yijiang Nan, Xiaojing Huang, Senior Member, IEEE, and Y. Jay Guo, Fellow, IEEE
}

\begin{abstract}
A generalized continuous wave synthetic aperture radar (GCW-SAR) concept is proposed in this paper. By using full-duplex (FD) radio frontend and continuous wave (CW) signalling, the GCW-SAR system can overcome a number of limitations inherent within existing SAR systems and achieve high resolution and wide swath remote sensing with low power signal transmission. Unlike the conventional pulsed SAR and the frequency modulated continuous wave SAR (FMCW-SAR), the GCW-SAR reconstructs a radar image by directly correlating the received one-dimensional raw data after self-interference cancellation (SIC) with predetermined location dependent reference signals. A fast imaging algorithm, called piecewise constant Doppler (PCD) algorithm, is also proposed, which produces the radar image recursively in the azimuth direction without any intermediate step, such as range compression and migration compensation, as required by conventional algorithms. By removing the stop-and-go assumption or slow time sampling in azimuth, the PCD algorithm not only achieves better imaging quality but also allows for more flexible waveform and system designs. Analyses and simulations show that the GCW-SAR tolerates significant self-interference and works well with a large selection of various system parameters. The work presented in this paper establishes a solid theoretical foundation for next generation imaging radars.
\end{abstract}

Index Terms-Synthetic aperture radar (SAR), full-duplex (FD), continuous wave (CW), high resultion and wide swath, frequency modulated continuous wave SAR (FMCW-SAR) and self-interference cancellation (SIC).

\section{INTRODUCTION}

$\mathbf{S}$ YNTHETIC aperture radar (SAR) is capable of generating high-resolution remote sensing images in all-day and allweather conditions and has been widely used in many civil and defense applications [1]-[3]. Mounted on a moving platform, SAR transmits a wideband signal and receives the echoes reflected from the observed targets. High range resolution can be obtained from the wide bandwidth of the transmitted signal, and high azimuth resolution relies on the Doppler frequency shift presented in the received signals due to the relative motion between platform and targets.

In a conventional pulsed SAR, the platform moves with a constant velocity and transmits a pulse train with appropriate pulse repetition frequency (PRF). It then receives and stores the echoes from the observed scene. During the reception

This work was supported under the Australian Research Council (ARC) Discovery Project DP 160101693.

Yijiang Nan, Xiaojing Huang, and Y. Jay Guo are with the Global Big Data Technologies Center, University of Technology Sydney, NSW, Australia. E-mails: Yijiang.Nan@student.uts.edu.au, Xiaojing.Huang@uts.edu.au, and Jay.Guo@uts.edu.au. mode, the transmission must be stopped to prevent interference to the received echoes. Switching between transmission and reception modes alternately not only makes the system operation complicated but also increases the peak transmission power [4]. In addition, the pulsed SAR system also suffers from some inherent weaknesses, such as the constraint on PRF selection [5]. On one hand, to achieve wider swath radar image, a lower PRF is required. On the other hand, to achieve higher azimuth resolution, the PRF should be higher. Such conflicting requirement known as the minimum antenna area constraint [5] renders it necessary to have a trade-off between azimuth resolution and swath width. Moreover, the PRF should be also properly selected to avoid the interference from the nadir reflectors as well as those with echo propagation delays longer than a pulse repetition interval due to the sidelobes of the transmission signal beam. Consequently, it is much more difficult for a conventional SAR to reconstruct radar images with both wider unambiguous swath and higher azimuth resolution.

A number of new SAR systems and novel imaging algorithms have been proposed over the past decades to solve the above mentioned problems. An idea solving the high peak transmission power problem is the combination of frequency modulated continuous wave (FMCW) signalling and SAR techniques, leading to a lightweight cost-effective imaging sensor, i.e., FMCW-SAR [4], [6], which can operate at a constant low transmission power. In the FMCW-SAR system, the radar transmits linearly frequency modulated (LFM) signal periodically, and then the received signal is mixed with a replica (reference signal) of the transmitted signal and downconverted to baseband to produce the beat signal via lowpass filtering. The frequency in the beat signal determines the range of a target. Although FMCW-SAR is a continuous wave (CW) system, it is nowadays processed like a pulsed SAR, adopting separate range compression and slow time sampling in azimuth, which introduces unnecessary azimuth ambiguities and range restrictions.

The classical methods dealing with the restrictions for achieving high resolution and wide swath are spotlight SAR and ScanSAR respectively. Spotlight SAR improves the azimuth resolution at the cost of noncontiguous coverage along the track [7], while ScanSAR achieves wider swath at the cost of impaired azimuth resolution [8]. In recent years, multipleinput and multiple-output (MIMO) technology has been applied to SAR imaging, and more degrees of freedom and improved spatial resolution are obtained from the advantages 
of MIMO architecture [9]-[15]. For example, displaced phase center antenna (DPCA) technique [12] is adopted to enable an efficient suppression of azimuth ambiguity that allows an unambiguous wider swath imaging. The digital beamforming technique with multiple receivers [13]-[15] separates the wide swath into multiple subswaths so that the echo delay difference is essentially reduced. Unfortunately, these techniques still adopt the stop-and-go assumption and the slow time in azimuth. Therefore, they cannot break the trade-off between azimuth resolution and range ambiguity.

In this paper, the concept of continuous wave imaging is revisited and a generalized continuous wave SAR (GCWSAR) is proposed for future high resolution remote sensing. The GCW-SAR is a combination of full-duplex (FD) radio technique and CW radar. In an FD system, transmission and reception are conducted at the same time in the same frequency band since the received signal can be extracted after selfinterference cancellation (SIC) [16]-[18]. Applying SIC to SAR system, both transmission and reception modes can work simultaneously and the image of an observed scene can be recovered from the received $\mathrm{CW}$ echo signal. Unlike the FMCW-SAR or conventional pulsed SAR, the slow time in azimuth for the separation of range and azimuth information is no longer adopted and various system restrictions are removed. The new system obtains only one-dimensional raw data by sampling the received echoes at an appropriate sampling rate according to the signal bandwidth and then retrieves the range and azimuth information jointly. Assuming the same radar sensitivity, the GCW-SAR will offer lots of potential advantages over conventional SARs. Firstly, transmitting CW signal retains the same advantages of low power operation as FMCW-SAR. Secondly, it breaks the trade-off between azimuth resolution and range ambiguity since the azimuth sampling rate is no longer swath dependent and can be as high as the range one (i.e., no slow time any more). Thirdly, jointly retrieving range and azimuth information removes some unnecessary intermediate steps in SAR signal processing such as range compression and migration. Moreover, the elimination of slow time in azimuth makes the transmitted waveform design much more flexible.

Self-interference cancellation can be generally achieved in three domains: propagation domain, analog-circuit domain, and digital domain [18]. To ensure simultaneous transmission and reception, the GCW-SAR system can adopt any or all of the appropriate SIC techniques. In propagation domain, if necessary, the system can use two separate antennas for transmission and reception respectively, and provide as high as possible isolation between transmitted and received signals through exploiting various signal propagation characteristics such as path-loss [19], [20], cross-polarization [21], [22], and antenna directionality [21], [22]. In analog-circuit domain, various cancellation techniques [23]-[25] can suppress selfinterference in the analog receive-chain circuitry before the receiver's analog-to-digital converter (ADC). In digital domain, the self-interference can be easily removed from the final reconstructed image since it represents a near-field reflection which is outside the transmission beam footprint. From the analysis and simulation results, the self-interference in digital domain has negligible impact on the imaging quality even at a signal-to-interference ratio (SIR) of $-45 d B$ in airborne case. Consequently, the only requirement for SIC in GCW-SAR is that the residual self-interference should be captured within the receiver's ADC dynamic range. For example, an ADC with 10 effective number of bits $(\mathrm{ENoB})$ can have a dynamic range of $60 \mathrm{~dB}$. A number of SIC techniques [19]-[25] have been proposed in recent years, and a total of $90 d B$ cancellation can be achieved in propagation and analog-circuit domains, which ensures that the GCW-SAR principle can be applied in airborne based SARs. For example, in a practical FMCW-SAR [6], the transmit power is $18 \mathrm{dBm}$, and the receiver noise floor is at $-90 \mathrm{dBm}$. The above mentioned $-45 d B$ SIR requirement implies that only $63 d B$ SIC is required before ADC. Considering the FM noise and other practical imperfection effects, more SIC is necessary especially for spaceborne SARs due to longer signal propagation and possibly higher transmit power. We believe that the increasingly better suppression in selfinterference will enable the implementation of such systems in the future.

Regarding imaging processing, the GCW-SAR in principle adopts the same time domain correlation or matched-filtering approach which is also known as back-projection [26]-[32]. However, unlike the back-projection algorithm (BPA) used in conventional SAR, the stop-and-go assumption or slow time concept is abandoned in GCW-SAR and the image is reconstructed from the one-dimensional raw data directly without the range compression as an intermediate step performed in slow time [26]. In doing that, the GCW-SAR system produces a set of reference signals over the aperture time based on the transmitted signal and the locations of the pixels to be imaged, and then focuses each image point by performing cross-correlation of the received signal with the corresponding reference signal. A piecewise linear approximation to the range between radar transmitter and target within the synthetic aperture is also adopted, leading to a complexity reduced algorithm, called piecewise constant Doppler (PCD) algorithm. Analysis and simulation demonstrate its superior performance over conventional methods. Note that this paper only describes the stripmap GCW-SAR (single-antenna, side-looking and constant antenna pointing direction) to illustrate the proposed principle. The other GCW-SAR modes will be studied in our future work.

The remainder of this paper is organized as follows. In Section II, the principle of GCW-SAR and the ideal matched filtering based one-dimensional data structure are introduced. The desired imaging ambiguity function is also analyzed accordingly. The PCD algorithm is proposed in Section III and its fast implementation and complexity analysis are also presented in details. Section IV shows the simulation results of GCW-SAR performance with the proposed PCD algorithm and provides the performance analyses and comparisons, including imaging qualities under various self-interference levels in digital domain and the impacts of the parameters used in PCD algorithm. Finally, Section V concludes this paper with a short discussion. 


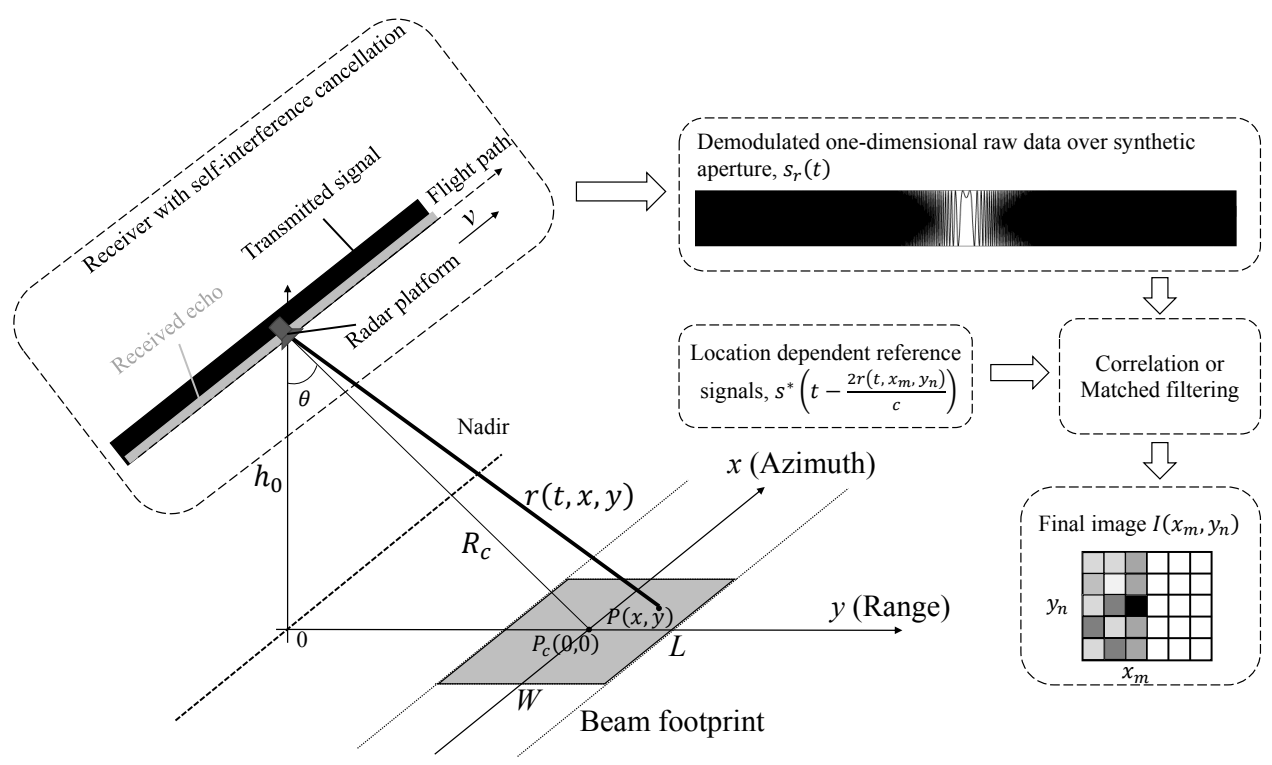

Fig. 1. GCW-SAR geometry and image reconstruction process in principle.

TABLE I

GCW-SAR PARAMETERS

\begin{tabular}{|c|c|}
\hline Symbols & Definitions \\
\hline$t$ & Time variable \\
\hline$R_{c}$ & Range from the antenna to the beam footprint center \\
\hline$r(t, x, y)$ & Slant range from the antenna to an arbitrary point $P(x, y)$ \\
\hline$\sigma(x, y)$ & Radar cross section (RCS) of point $P(x, y)$ \\
\hline$c, v$ & Speeds of light and radar platform \\
\hline$T$ & Traveling time over the synthetic aperture \\
\hline$T_{r}$ & Transmitted chirp signal repetition interval \\
\hline$T_{s}$ & Sampling interval of received signal \\
\hline$W, L$ & Range and azimuth widths of the beam footprint respectively \\
\hline$W_{a}, L_{a}$ & Height and width of the antenna aperture \\
\hline$\Delta x, \Delta y$ & Pixel spacings in $x$ and $y$ axies respectively \\
\hline$\theta$ & Beam incident angle \\
\hline$\lambda, f_{c}$ & Transmit carrier wavelength and frequency respectively \\
\hline$\delta_{x}, \delta_{y}$ & Azimuth and range resolutions respectively \\
\hline
\end{tabular}

\section{GCW-SAR PRINCIPLE}

In this section, the geometry of a stripmap GCW-SAR is presented, followed by the imaging algorithm description and ambiguity function analysis.

\section{A. System Geometry}

The mathematical symbols and their definitions used in this paper are given in Table I. The GCW-SAR system geometry and image reconstruction process are illustrated in Fig. 1. The radar, at a height $h_{0}$, travels at a constant speed $v$ in the $x$-direction and the origin $(0,0)$ is located at the center of the beam footprint, which is illustrated as the shaded area. The coordinate of an arbitrary point $P$ in the beam footprint is $(x, y)$ in Fig. 1. The round-trip delay time for the wave propagation varies as a function of time $t$. For simplicity, the difference between forward and back trips can be negligible [33] and the instantaneous slant range is expressed as

$$
r(t, x, y)=\sqrt{\left(R_{c} \sin \theta+y\right)^{2}+(x-v t)^{2}+h_{0}^{2}}
$$

where $t \in\left(\frac{x}{v}-\frac{T}{2}, \frac{x}{v}+\frac{T}{2}\right)$ and $T$ is equal to $L / v$.

In a GCW-SAR, the baseband transmitted waveform $s(t)$ is up-converted to the carrier frequency and transmitted continuously. After self-interference cancellation, the received raw GCW-SAR data can be represented as a one-dimensional continuous wave signal $s_{r}(t)$, which is a superposition of a large number of reflected echoes from the beam footprint, i.e.,

$$
\begin{aligned}
s_{r}(t)= & \int_{v t-\frac{L}{2}}^{v t+\frac{L}{2}} \int_{-\frac{W}{2}}^{\frac{W}{2}} \sigma(x, y) s\left(t-\frac{2 r(t, x, y)}{c}\right) \\
& e^{-j \frac{4 \pi}{\lambda} r(t, x, y)} d y d x
\end{aligned}
$$

where the center of beam footprint is $(0, v t)$ and $\sigma(x, y)$ is the radar cross section (RCS) of the image point $(x, y)$. For simplicity, we only consider the flat terrain in this paper. The non-flat terrain may lead to the height-incurred error [13]. In conventional SAR, this problem can be resolved by using antenna array on receiver with digital beamforming technique [13], which can be also applied to GCW-SAR. Further threedimensional GCW-SAR imaging will be studied in our future work.

\section{B. Image Reconstruction}

Following the stop-and-go assumption and the slow time sampling in azimuth, the conventional SAR image reconstruction is generally made of two separate range and azimuth compressions. Such imaging processing is not suitable in GCW-SAR, since the one-dimensional raw data involve both range and azimuth modulations which cannot be separated over longer pulse repetition interval for wider swath imaging. Therefore, both time delay and Doppler frequency shift of the received raw data should be considered jointly during the imaging process.

In the context of GCW-SAR, the ideal image reconstruction process from the one-dimensional raw data is briefly described 
as follows. The complexity-reduced algorithm will be presented in details in the next section.

For an arbitrary point $P\left(x_{m}, y_{n}\right)$ in the transmitted signal beam footprint, GCW-SAR produces a location dependent reference signal and then recovers the RCS, $\sigma\left(x_{m}, y_{n}\right)$, by passing the received signal through a matched filter, which is expressed as

$$
\begin{aligned}
I\left(x_{m}, y_{n}\right)= & \int_{\frac{x_{m}}{v}-\frac{T}{2}}^{\frac{x_{m}}{v}+\frac{T}{2}} s_{r}(t) s^{*}\left(t-\frac{2 r\left(t, x_{m}, y_{n}\right)}{c}\right) \\
& e^{j \frac{4 \pi}{\lambda} r\left(t, x_{m}, y_{n}\right)} d t
\end{aligned}
$$

where $I\left(x_{m}, y_{n}\right)$ is the output of the matched filter, the asterisk * indicates complex conjugation and $r\left(t, x_{m}, y_{n}\right)$ indicates the range from the radar antenna to the point $\left(x_{m}, y_{n}\right)$. Based on (3), the image reconstruction process in principle can be described as follows. Firstly, assuming that the beam footprint is filled with a set of uniformly distributed point scatterers, whose spacings along $x$ and $y$ axises are $\Delta x$ and $\Delta y$ respectively, the pixels in the final image are arranged as a twodimensional matrix. Secondly, the expected received signals from these pixels are used as the location dependent reference signals to be used in the matched filters. Thirdly, after the radar collects all the raw data over one synthetic aperture distance in azimuth direction, a set of pixel values in range direction for a given azimuth coordinate can be obtained by performing correlation between the received signal and the corresponding reference signals. Finally, as the GCW-SAR moves on in the azimuth direction, consecutive sets of pixel values in range direction can be obtained and a two dimensional image can be finally reconstructed. This process is also shown in Fig. 1.

\section{Ambiguity Function}

Further analysis of (3) can be performed to relate the image output to an ambiguity function which is defined as a twodimensional function showing a distorted image of a point target [34], [35]. Substitution of (2) into (3) gives

$$
\begin{aligned}
I\left(x_{m}, y_{n}\right)= & \int_{\frac{x_{m}}{v}-\frac{T}{2}}^{\frac{x_{m}}{v}+\frac{T}{2}} \int_{v t-\frac{L}{2}}^{v t+\frac{L}{2}} \int_{-\frac{W}{2}}^{\frac{W}{2}} \sigma(x, y) s\left(t-\frac{2 r(t, x, y)}{c}\right) \\
& \cdot s^{*}\left(t-\frac{2 r\left(t, x_{m}, y_{n}\right)}{c}\right) e^{j \frac{4 \pi}{\lambda}\left(r\left(t, x_{m}, y_{n}\right)-r(t, x, y)\right)} \\
& \cdot d y d x d t \\
= & \int_{x_{m}-L}^{x_{m}+L} \int_{-\frac{W}{2}}^{\frac{W}{2}} A_{x, y}\left(x_{m}, y_{n}\right) \sigma(x, y) d y d x
\end{aligned}
$$

where

$$
\begin{aligned}
& A_{x, y}\left(x_{m}, y_{n}\right) \\
& =\int_{\frac{x_{m}}{v}-\frac{T}{2}}^{\frac{x_{m}}{v}+\frac{T}{2}} s\left(t-\frac{2 r(t, x, y)}{c}\right) s^{*}\left(t-\frac{2 r\left(t, x_{m}, y_{n}\right)}{c}\right) \\
& \cdot e^{j \frac{4 \pi}{\lambda}\left(r\left(t, x_{m}, y_{n}\right)-r(t, x, y)\right)} d t
\end{aligned}
$$

is the ambiguity function of the point $(x, y)$, which can be considered as a weighting function on the $\operatorname{RCS} \sigma(x, y)$. The output of the matched filter is actually the weighted average of RCS over two consecutive beam footprints along azimuth direction.

With the slow time sampling in azimuth, the range and azimuth resolutions in conventional SARs have been derived in the literature [34]. However, under the one-dimensional data structure without slow time involved, the derivation of the range and azimuth resolutions requires a different approach, which is provided as follows.

Firstly, the slant range defined in (1) can be approximated as

$$
\begin{aligned}
r & =\sqrt{R(y)^{2}+(x-v t)^{2}} \approx R(y)+\frac{(x-v t)^{2}}{2 R(y)}, \\
t & \in\left[\frac{x}{v}-\frac{T}{2}, \frac{x}{v}+\frac{T}{2}\right]
\end{aligned}
$$

where $R(y)=\sqrt{\left(R_{c} \sin \theta+y\right)^{2}+h_{0}}$, which is valid when $|x-v t| \ll R(y)$ is satisfied.

Since the received echoes are the far-field reflections, and the slant range for any pixel satisfies the condition $r\left(t+\frac{x_{m}}{v}, x_{m}, y_{n}\right)=r\left(t, 0, y_{n}\right)$, the ambiguity function of an arbitrary point $\left(x_{m}, y_{n}\right)$ can be related to the one of the origin $(0,0)$ as $A_{x_{m}, y_{n}}(x, y) \approx A_{0,0}\left(x-x_{m}, y-y_{n}\right)$. For simplicity, we only consider the ambiguity function at the origin $(0,0)$ which can be expressed as

$$
\begin{aligned}
& A_{0,0}(x, y) \\
& =\int_{-\frac{T}{2}}^{\frac{T}{2}} s\left(t-\frac{2 r(t, 0,0)}{c}\right) s^{*}\left(t-\frac{2 r(t, x, y)}{c}\right) \\
& \cdot e^{j \frac{4 \pi}{\lambda}(r(t, x, y)-r(t, 0,0))} d t \\
& \approx \int_{-\frac{T}{2}}^{\frac{T}{2}} s\left(t+\frac{2(r(t, x, y)-r(t, 0,0))}{c}\right) s^{*}(t) \\
& \cdot e^{j \frac{4 \pi}{\lambda}(r(t, x, y)-r(t, 0,0))} d t .
\end{aligned}
$$

It is apparent that the ambiguity function closely relates to the range difference $r(t, x, y)-r(t, 0,0)$. In this section, two cases of the ambiguity function $A_{0,0}(0, y)$ and $A_{0,0}(x, 0)$ are considered respectively: the first one indicates the range resolution and the second one indicates the azimuth resolution.

1) Range resolution: When $x=0, r(t, 0, y)-r(t, 0,0) \approx$ $\left(R(y)-R_{c}\right)\left(1-\frac{(v t)^{2}}{2 R(y) R_{c}}\right) \approx R(y)-R_{c}$ since the term $\frac{(v t)^{2}}{2 R(y) R_{c}}$ can be neglected as $R_{c}>>L$. Eq. (7) can then be expressed as

$$
\begin{aligned}
& A_{0,0}(0, y) \\
& \approx e^{j \frac{4 \pi}{\lambda}\left(R(y)-R_{c}\right)} \int_{-\frac{T}{2}}^{\frac{T}{2}} s\left(t+\frac{2\left(R(y)-R_{c}\right)}{c}\right) s^{*}(t) d t .
\end{aligned}
$$

Assuming that $s(t)$ is any normalized low pass baseband signal, $\int_{-\frac{T}{2}}^{\frac{T}{2}}|s(t)|^{2} d t=1$, with constant energy spectrum in its bandwidth $B$, its auto-correlation function will be a sinc function. Consequently, Eq. (8) can be further expressed as

$$
A_{0,0}(0, y) \approx e^{j \frac{4 \pi}{\lambda}\left(R(y)-R_{c}\right)} \frac{\sin \left(\pi \frac{2 B}{c}\left(R(y)-R_{c}\right)\right)}{\pi \frac{2 B}{c}\left(R(y)-R_{c}\right)} .
$$

The range resolution $\delta_{y}$ is determined by $R(y)-R_{c}=\frac{c}{2 B}$ which is the first null point in the ambiguity function. Given that the beam incident angle is $\theta$, we have

$$
\delta_{y}=\frac{c}{2 B \sin \theta} \text {. }
$$


2) Azimuth resolution: When $y=0$, the range difference can be approximated as $r(t, x, 0)-r(t, 0,0) \approx \frac{x^{2}-2 v x t}{2 R_{c}}$ and the ambiguity function can be expressed as

$$
\begin{aligned}
& A_{0,0}(x, 0) \approx \int_{-\frac{T}{2}}^{\frac{T}{2}} e^{j \frac{4 \pi}{\lambda} \frac{x^{2}-2 v x t}{2 R_{c}}} s\left(t+\frac{x^{2}-2 v x t}{c R_{c}}\right) s^{*}(t) d t \\
& =\int_{-\frac{T}{2}}^{\frac{T}{2}} e^{j \frac{4 \pi}{\lambda} \frac{x^{2}-2 v x t}{2 R_{c}}} s\left(t+\frac{1}{f_{c}} \frac{x^{2}-2 v x t}{\lambda R_{c}}\right) s^{*}(t) d t
\end{aligned}
$$

where $f_{c}=c / \lambda$ is the carrier frequency. Assuming that $s(t)$ is a normalized phase only signal, $s(t)=\frac{1}{\sqrt{T}} e^{j \phi(t)}$, Eq. (11) can be simplified as

$$
\begin{aligned}
& A_{0,0}(x, 0) \\
& =\frac{1}{T} \int_{-\frac{T}{2}}^{\frac{T}{2}} e^{j \frac{4 \pi}{\lambda} \frac{x^{2}-2 v x t}{2 R_{c}}} e^{j\left(\phi\left(t+\frac{1}{f_{c}} \frac{x^{2}-2 v x t}{\lambda R_{c}}\right)-\phi(t)\right)} d t \\
& \approx \frac{1}{T} \int_{-\frac{T}{2}}^{\frac{T}{2}} e^{j 2 \pi \frac{x^{2}-2 v x t}{\lambda R_{c}}} e^{j \phi^{\prime}(t) \frac{1}{f_{c}} \frac{x^{2}-2 v x t}{\lambda R_{c}}} d t \\
& =\frac{1}{T} \int_{-\frac{T}{2}}^{\frac{T}{2}} e^{j 2 \pi \frac{x^{2}-2 v x t}{\lambda R_{c}}\left(1+\frac{\phi^{\prime}(t)}{2 \pi f_{c}}\right)} d t \\
& \approx \frac{1}{T} \int_{-\frac{T}{2}}^{\frac{T}{2}} e^{j 2 \pi \frac{x^{2}-2 v x t}{\lambda R_{c}}} d t=e^{j \frac{2 \pi x^{2}}{\lambda R_{c}}} \frac{\sin \left(\pi \frac{2 L}{\lambda R_{c}} x\right)}{\pi \frac{2 L}{\lambda R_{c}} x}
\end{aligned}
$$

where $\phi^{\prime}(t)$ is the first order derivative of $\phi(t)$. Because $2 \pi f_{c}$ is far larger than the maximum $\phi^{\prime}(t)$ which is less than $2 \pi B$, $\frac{\phi^{\prime}(t)}{2 \pi f_{c}}$ is neglected in deriving (12). The azimuth resolution $\delta_{x}$ is determined by $x=\frac{\lambda R_{c}}{2 L}$ which is the first null point in the ambiguity function and hence

$$
\delta_{x}=\frac{\lambda R_{c}}{2 L}=\frac{L_{a}}{2}
$$

where $\lambda R_{c} / L$ is equal to the antenna aperture $L_{a}$.

Though the range and azimuth resolutions of the GCW-SAR are the same as those of the conventional pulsed SAR and FMCW-SAR respectively, the above analysis clearly shows the requirements of the transmitted signal, i.e., a constant energy spectrum in the frequency domain and a constant signal envelope in the time domain, under which the range and azimuth resolutions are bounded by (10) and (13) respectively. Note that in a conventional pulsed SAR and FMCW-SAR, the transmitted signals must be a pulse train and a continuous periodic $\mathrm{CW}$ with a carefully selected PRF respectively, whereas for the GCW-SAR, the transmitted signal does not have to be periodic. This enables great flexibility in signal waveform design. With noise radar technologies, a noise waveform can be also used as the transmitted signal [36]. However, it clearly does not satisfy all the above mentioned requirements and hence cannot achieve the optimal range and azimuth resolutions.

\section{Piecewise CONSTANT Doppler Algorithm}

With the general image reconstruction method described in section II.B, integration over the entire synthetic aperture time is necessary for each pixel in the GCW-SAR image. As the amount of pixels in a high resolution and wide swath image will be significant, the direct implementation of the algorithm leads to a great computational complexity. However, existing fast imaging algorithms designed for BPAs [26]-[32] are not applicable to GCW-SAR, since they depend on the two-dimensional raw data structure and also involve slow time sampling in azimuth under the stop-and-go assumption. In this section, we analyze the relationships between the correlations of the adjacent pixels in azimuth direction and design a complexity reduced approach which calculates the correlation recursively after applying piecewise linear approximation to the range curve.

\section{A. Principle}

The time delay of the received signal from an imaging point $(x, y)$ changes in response to its slant range $r(t, x, y)$. Assuming that the platform is moving at a constant speed in a straight line, the variation of the slant range is equal to a parabola. In order to deal with this nonlinear range curve, the parabola can be divided into multiple linear segments linked end to end. Therefore, the Doppler frequency shift in each segment can be considered as a constant. According to (3), the image $I\left(x_{m}, y_{n}\right)$ of the pixel $\left(x_{m}, y_{n}\right)$ involves integration over an entire synthetic aperture time $\left[-\frac{T}{2}+\frac{x_{m}}{v}, \frac{T}{2}+\frac{x_{m}}{v}\right]$. If we divide the time interval $\left[-\frac{T}{2}, \frac{T}{2}\right]$ into $P$ segments and define the time instants at the two ends of the interval as well as the segment joint points as $t_{p}=p \frac{T}{P}$ for $p=-\frac{P}{2},-\frac{P}{2}+1, \ldots, \frac{P}{2}$, the $p$ th segment of the synthetic aperture time can be denoted as $\left(\frac{x_{m}}{v}+t_{p}, \frac{x_{m}}{v}+t_{p+1}\right]$ and the corresponding correlation value over this time interval can be calculated as

$$
\begin{aligned}
& I_{p}\left(x_{m}, y_{n}\right) \\
& =\int_{\frac{x_{m}}{v}+t_{p}}^{\frac{x_{m}}{v}+t_{p+1}} s_{r}(t) s^{*}\left(t-\frac{2 r\left(t, x_{m}, y_{n}\right)}{c}\right) e^{j \frac{4 \pi}{\lambda} r\left(t, x_{m}, y_{n}\right)} d t .
\end{aligned}
$$

The image $I\left(x_{m}, y_{n}\right)$ of the pixel $\left(x_{m}, y_{n}\right)$ is thus expressed as

$$
I\left(x_{m}, y_{n}\right)=\sum_{p=-\frac{P}{2}}^{\frac{P}{2}-1} I_{p}\left(x_{m}, y_{n}\right)
$$

Now, we derive the correlation value $I_{p}\left(x_{m}+\Delta x, y_{n}\right)$ over the $p$ th segment of a synthetic aperture time for the image point $\left(x_{m}+\Delta x, y_{n}\right)$ in relation to $I_{p}\left(x_{m}, y_{n}\right)$. In this segment, the received echo from the image point $\left(x_{m}+\Delta x, y_{n}\right)$ is made up of two parts: the first one is received during the time interval $\left(\frac{x_{m}}{v}+\frac{\Delta x}{v}+t_{p}, \frac{x_{m}}{v}+t_{p+1}\right]$ which is also used to calculate $I_{p}\left(x_{m}, y_{n}\right)$; the second one is received during the time interval $\left(\frac{x_{m}}{v}+t_{p+1}, \frac{x_{m}}{v}+\frac{\Delta x}{v}+t_{p+1}\right]$. Since $r\left(t+\frac{x}{v}, x, y\right)=r(t, 0, y)$, 


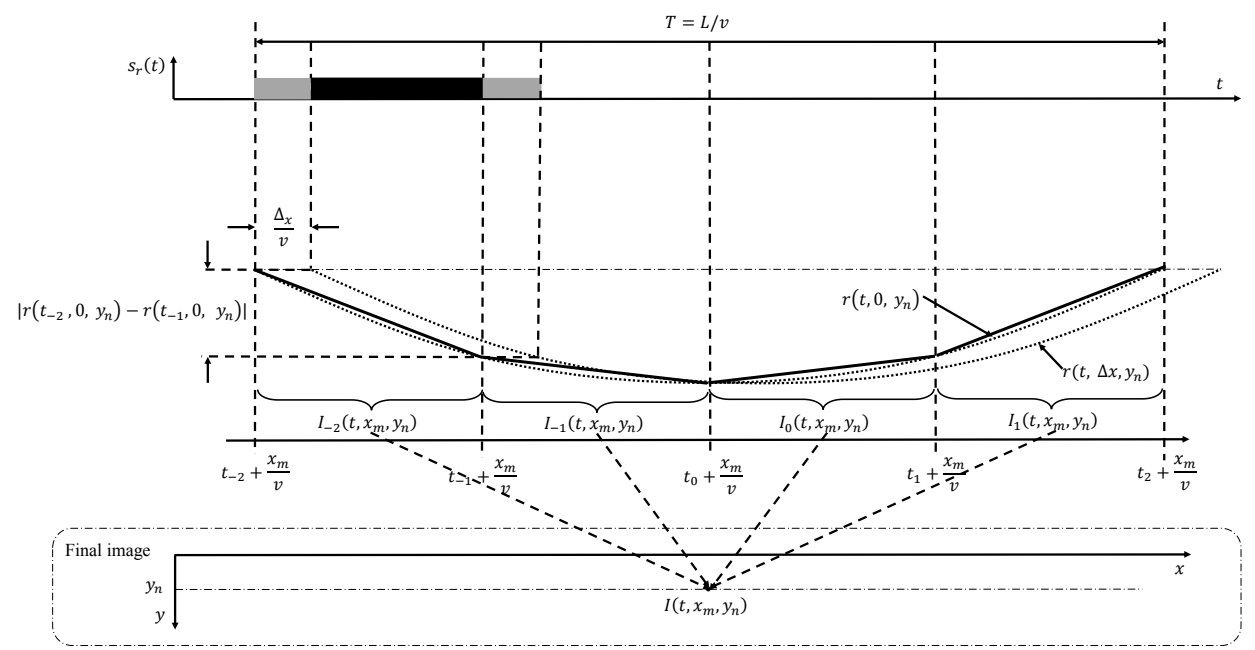

Fig. 2. Segmentation, linearization and their relationship with image reconstruction.

the image $I_{p}\left(x_{m}+\Delta x, y_{n}\right)$ can be expressed as

$$
\begin{aligned}
& I_{p}\left(x_{m}+\Delta x, y_{n}\right) \\
= & \int_{\frac{x_{m}}{v}+\frac{\Delta x}{v}+t_{p}}^{\frac{x_{m}}{v}+t_{p+1}} s_{r}(t) s^{*}\left(t-\frac{2 r\left(t, x_{m}+\Delta x, y_{n}\right)}{c}\right) \\
& \cdot e^{j \frac{4 \pi}{\lambda} r\left(t, x_{m}+\Delta x, y_{n}\right)} d t \\
& +\int_{\frac{x_{m}}{v}+t_{p+1}}^{\frac{x}{v}+\frac{\Delta x}{v}+t_{p+1}} s_{r}(t) s^{*}\left(t-\frac{2 r\left(t, x_{m}+\Delta x, y_{n}\right)}{c}\right) \\
& \cdot e^{j \frac{4 \pi}{\lambda} r\left(t, x_{m}+\Delta x, y_{n}\right)} d t \\
= & \int_{\frac{\Delta x}{v}+t_{p}}^{t_{p+1}} s_{r}\left(t+\frac{x_{m}}{v}\right) s^{*}\left(t+\frac{x_{m}}{v}-\frac{2 r\left(t, \Delta x, y_{n}\right)}{c}\right) \\
& \cdot e^{j \frac{4 \pi}{\lambda} r\left(t, \Delta x, y_{n}\right)} d t \\
& +\int_{t_{p+1}}^{\frac{\Delta x}{v}+t_{p+1}} s_{r}\left(t+\frac{x_{m}}{v}\right) s^{*}\left(t+\frac{x_{m}}{v}-\frac{2 r\left(t, \Delta x, y_{n}\right)}{c}\right) \\
& \cdot e^{j \frac{4 \pi}{\lambda} r\left(t, \Delta x, y_{n}\right)} d t .
\end{aligned}
$$

The slant range $r\left(t, \Delta x, y_{n}\right)$ in the $p$ th segment can be approximated as a linear function of $t$ as

$$
\begin{aligned}
& r\left(t, \Delta x, y_{n}\right) \\
& \approx r\left(t_{p}+\frac{\Delta x}{v}, \Delta x, y_{n}\right) \\
& +\frac{r\left(t_{p+1}+\frac{\Delta x}{v}, \Delta x, y_{n}\right)-r\left(t_{p}+\frac{\Delta x}{v}, \Delta x, y_{n}\right)}{t_{p+1}-t_{p}}\left(t-t_{p}-\frac{\Delta x}{v}\right) \\
& =r\left(t_{p}, 0, y_{n}\right)-\frac{\lambda}{2} f_{D_{p}}\left(y_{n}\right)\left(t-t_{p}-\frac{\Delta x}{v}\right) \\
& =r\left(t, 0, y_{n}\right)+\frac{\lambda}{2} f_{D_{p}}\left(y_{n}\right) \frac{\Delta x}{v}
\end{aligned}
$$

where $f_{D_{p}}\left(y_{n}\right)=-\frac{2}{\lambda} \frac{r\left(t_{p+1}, 0, y_{n}\right)-r\left(t_{p}, 0, y_{n}\right)}{t_{p+1}-t_{p}}$ is defined as the constant Doppler frequency shift for segment $p$. For the same $R\left(y_{n}\right)$, the time delay difference in the baseband reference signal between two adjacent pixels $\left(x_{m}, y_{n}\right)$ and $\left(x_{m}+\Delta x, y_{n}\right)$ can be neglected since $|x-v t|<<R\left(y_{n}\right)$, indicating $s^{*}\left(t+\frac{x_{m}}{v}-\frac{2 r\left(t, \Delta x, y_{n}\right)}{c}\right) \approx s^{*}\left(t+\frac{x_{m}}{v}-\frac{2 r\left(t, 0, y_{n}\right)}{c}\right)$. Eq. (16) thus can be re-written as

$$
\begin{aligned}
& I_{p}\left(x_{m}+\Delta x, y_{n}\right) \\
& =\int_{t_{p}}^{t_{p+1}} s_{r}\left(t+\frac{x_{m}}{v}\right) s^{*}\left(t+\frac{x_{m}}{v}-\frac{2 r\left(t, \Delta x, y_{n}\right)}{c}\right) \\
& \cdot e^{j \frac{4 \pi}{\lambda} r\left(t, \Delta x, y_{n}\right)} d t \\
& -\int_{t_{p}}^{\frac{\Delta x}{v}+t_{p}} s_{r}\left(t+\frac{x_{m}}{v}\right) s^{*}\left(t+\frac{x_{m}}{v}-\frac{2 r\left(t, \Delta x, y_{n}\right)}{c}\right) \\
& \text { - } e^{j \frac{4 \pi}{\lambda} r\left(t, \Delta x, y_{n}\right)} d t \\
& +\int_{t_{p+1}}^{\frac{\Delta x}{v}+t_{p+1}} s_{r}\left(t+\frac{x_{m}}{v}\right) s^{*}\left(t+\frac{x_{m}}{v}-\frac{2 r\left(t, \Delta x, y_{n}\right)}{c}\right) \\
& \cdot e^{j \frac{4 \pi}{\lambda} r\left(t, \Delta x, y_{n}\right)} d t \\
& =\int_{t_{p}}^{t_{p+1}} s_{r}\left(t+\frac{x_{m}}{v}\right) s^{*}\left(t+\frac{x_{m}}{v}-\frac{2 r\left(t, 0, y_{n}\right)}{c}\right) \\
& \cdot e^{j \frac{4 \pi}{\lambda}\left(r\left(t, 0, y_{n}\right)+\frac{\lambda}{2} f_{D_{p}}\left(y_{n}\right) \frac{\Delta x}{v}\right)} d t \\
& -\int_{t_{p}}^{\frac{\Delta x}{v}+t_{p}} s_{r}\left(t+\frac{x_{m}}{v}\right) s^{*}\left(t+\frac{x_{m}}{v}-\frac{2 r\left(t, \Delta x, y_{n}\right)}{c}\right) \\
& \cdot e^{j \frac{4 \pi}{\lambda} r\left(t, \Delta x, y_{n}\right)} d t \\
& +\int_{t_{p+1}}^{\frac{\Delta x}{v}+t_{p+1}} s_{r}\left(t+\frac{x_{m}}{v}\right) s^{*}\left(t+\frac{x_{m}}{v}-\frac{2 r\left(t, \Delta x, y_{n}\right)}{c}\right) \\
& \cdot e^{j \frac{4 \pi}{\lambda} r\left(t, \Delta x, y_{n}\right)} d t \\
& =I_{p}\left(x_{m}, y_{n}\right) e^{j 2 \pi f_{D_{p}}\left(y_{n}\right) \frac{\Delta x}{v}}-\int_{0}^{\frac{\Delta x}{v}} s_{r}\left(t+t_{p}+\frac{x_{m}}{v}\right) \\
& \cdot s^{*}\left(t+t_{p}+\frac{x_{m}}{v}-\frac{2 r\left(t+t_{p}, \Delta x, y_{n}\right)}{c}\right) e^{j \frac{4 \pi}{\lambda} r\left(t+t_{p}, \Delta x, y_{n}\right)} d t \\
& +\int_{0}^{\frac{\Delta x}{v}} s_{r}\left(t+t_{p+1}+\frac{x_{m}}{v}\right) \\
& \cdot s^{*}\left(t+t_{p+1}+\frac{x_{m}}{v}-\frac{2 r\left(t+t_{p+1}, \Delta x, y_{n}\right)}{c}\right) \\
& \text { - } e^{j \frac{4 \pi}{\lambda} r\left(t+t_{p+1}, \Delta x, y_{n}\right)} d t \text {. }
\end{aligned}
$$




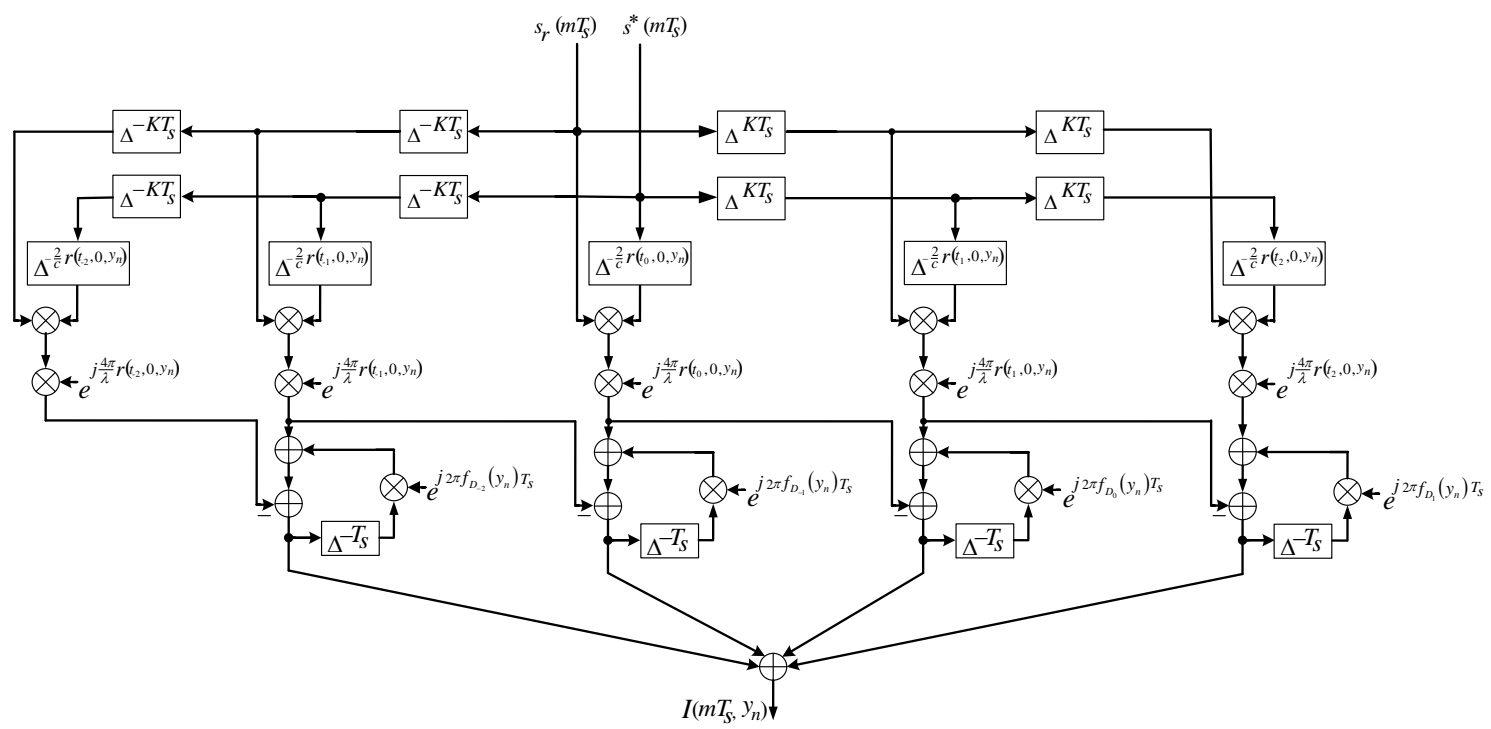

Fig. 3. Flow graph of PCD implementation for a given $y_{n}$, where the $\Delta^{ \pm \tau}$ denotes time delay or advance by $\tau$.

Taking $P=4$ as an example, the segmentation, linearization and their relationship with the image reconstruction are shown in Fig. 2. The dotted curves indicate the slant ranges of the pixels $\left(x_{m}, y_{n}\right)$ and $\left(x_{m}+\Delta x, y_{n}\right)$ respectively, which are approximated as the solid linear segments. In any segment $p$, the recursive imaging process is explained as follows:

1) Assume that the correlation $I_{p}\left(x_{m}, y_{n}\right)$ in the interval $\left(t_{p}+\frac{x_{m}}{v}, t_{p+1}+\frac{x_{m}}{v}\right]$ has been obtained for the pixel $\left(x_{m}, y_{n}\right)$. To calculate the correlation in the integration interval $\left(t_{p}+\frac{x_{m}}{v}+\frac{\Delta x}{v}, t_{p+1}+\frac{x_{m}}{v}+\frac{\Delta x}{v}\right]$ for the pixel $\left(x_{m}+\right.$ $\left.\Delta x, y_{n}\right)$, we first compensate for the Doppler frequency shift in the previously calculated $I_{p}\left(x_{m}, y_{n}\right)$, which is achieved by multiplying $I_{p}\left(x_{m}, y_{n}\right)$ by $e^{j 2 \pi f_{D_{p}}\left(y_{n}\right) \frac{\Delta x}{v}}$.

2) The second step is to remove the correlation obtained in the interval $\left(t_{p}+\frac{x_{m}}{v}, t_{p}+\frac{x_{m}}{v}+\frac{\Delta x}{v}\right]$ for the pixel $\left(x_{m}, y_{n}\right)$.

3) Finally, add new signal correlation during the interval $\left(t_{p+1}+\frac{x_{m}}{v}, t_{p+1}+\frac{x_{m}}{v}+\frac{\Delta x}{v}\right]$ to obtain $I_{p}\left(x_{m}+\Delta x, y_{n}\right)$.

\section{B. Implementation}

To ensure the quality of the final image, we set the number of pixels to $N=\frac{2 W}{\delta_{y}} /$ and $M=\frac{L}{T_{s} v}$ in range and azimuth respectively. After the sampling of the demodulated received signal, the data sequence is represented as $s_{r}\left(m T_{s}\right)$ and the coordinate of the pixel $\left(x_{m}, y_{n}\right)$ can be rewritten as $\left(m T_{s} v, y_{n}\right)$. The number of samples of the received signal in each segment is set to $K$, so that $t_{p+1}=t_{p}+K T_{s}$.

Based on (18) and letting $\Delta x=T_{s} v$, the corresponding recursive process is further derived as

$$
\begin{aligned}
& I_{p}\left((m+1) T_{s} v, y_{n}\right) \\
& =I_{p}\left(m T_{s} v, y_{n}\right) e^{j 2 \pi f_{D_{p}}\left(y_{n}\right) T_{s}}-T_{s} s_{r}\left(m T_{s}+t_{p}+T_{s}\right) \\
& \cdot s^{*}\left(m T_{s}+t_{p}+T_{s}-\frac{2 r\left(t_{p}+T_{s}, T_{s} v, y_{n}\right)}{c}\right) \\
& \cdot e^{j \frac{4 \pi}{\lambda} r\left(t_{p}+T_{s}, T_{s} v, y_{n}\right)}+T_{s} s_{r}\left(m T_{s}+t_{p+1}+T_{s}\right) \\
& \cdot s^{*}\left(m T_{s}+t_{p+1}+T_{s}-\frac{2 r\left(t_{p+1}+T_{s}, T_{s} v, y_{n}\right)}{c}\right) \\
& \cdot e^{j \frac{4 \pi}{\lambda} r\left(t_{p+1}+T_{s}, T_{s} v, y_{n}\right)} \\
& =I_{p}\left(m T_{s} v, y_{n}\right) e^{j 2 \pi f_{D_{p}}\left(y_{n}\right) T_{s}}-T_{s} s_{r}\left((m+1) T_{s}+t_{p}\right) \\
& \cdot s^{*}\left((m+1) T_{s}+t_{p}-\frac{2 r\left(t_{p}, 0, y_{n}\right)}{c}\right) e^{j \frac{4 \pi}{\lambda} r\left(t_{p}, 0, y_{n}\right)} \\
& +T_{s} s_{r}\left((m+1) T_{s}+t_{p+1}\right) \\
& \cdot s^{*}\left((m+1) T_{s}+t_{p+1}-\frac{2 r\left(t_{p+1}, 0, y_{n}\right)}{c}\right) \\
& \cdot e^{j \frac{4 \pi}{\lambda} r\left(t_{p+1}, 0, y_{n}\right)} .
\end{aligned}
$$

Taking $P=4$ as an example, the flow graph of the PCD implemention is shown in Fig. 3. We see that, instead of performing correlation for each pixel individually, the PCD algorithm updates the correlation recursively and thus saves much data storage and reduces the computational complexity.

\section{Complexity}

Existing SAR imaging algorithms come in two broad classes: frequency domain methods, e.g., range Doppler algorithm (RDA), and back-projection algorithms. For the sake of comparison, an $N \times N$ final image is reconstructed by using RDA, conventional BPAs and PCD algorithm respectively.

The RDA adopts range and azimuth compressions with range cell migration compensation (RCMC). The numbers of complex multiplications required for one-dimensional fast Fourier transform (FFT) are $\mathrm{N} \log _{2} N$ [37]. Hence, the range 


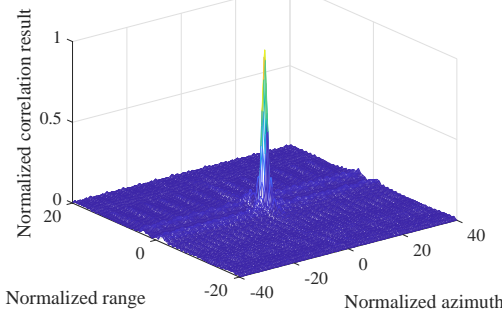

(a)

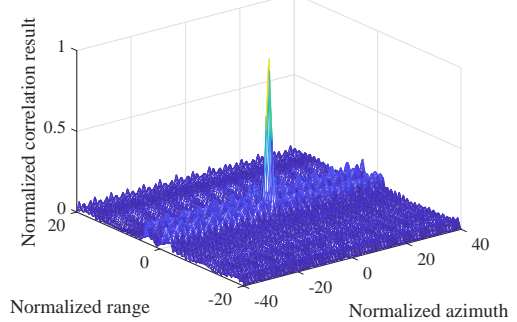

(b)

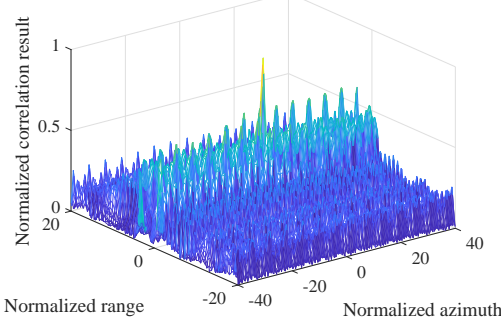

(c)

Fig. 4. SIR performance in an airborne GCW-SAR: (a), (b), and (c) show the GCW-SAR images of a point scatterer with PCD algorithm at SIR=-45dB, $-55 d B$, and $-65 d B$ respectively.

and azimuth compressions need totally $4 N^{2} \log _{2} N+2 N^{2}$ complex multiplications, including FFT and inverse FFT. Supposing that the number of complex multiplications required to interpolate one data sample is $N_{\text {interp }}$, the total number of complex multiplications required for the RDA is $4 N^{2} \log _{2} N+N^{2} \cdot\left(N_{\text {interp }}+2\right)$.

The conventional BPA reconstructs each pixel of the SAR image individually from the range compressed SAR data. The RCS of each image pixel can be obtained by the correlation over the synthetic aperture, thus the azimuth compression consists of $N$ complex multiplications. Considering the interpolation of data, the total number of complex multiplications required to reconstruct an $N \times N$ final image is $2 N^{2} \log _{2} N+N^{3}+N^{2} \cdot\left(N_{\text {interp }}+1\right)$.

The PCD algorithm updates the correlation recursively. After the correlation for the first pixel, each pixel in azimuth only needs $3 P+2$ complex multiplications as shown in Fig. 3 . Considering that GCW-SAR adopts the CW transmit signal, the number of complex multiplications required to reconstruct an $N \times N$ final image is $(3 P+2) \cdot N^{2}$. Note that the $N \times N$ final image is much smaller in terms of physical area than that of pulsed SAR due to the sample-by-sample recursion. To reconstruct the image of the same area, the GCW-SAR system requires $(3 P+2) \cdot N^{3}$ complex multiplications assuming that one synthetic aperture time has $N^{2}$ samples. However, a decimated PCD algorithm which performs recursion over multiple samples can be developed to reduce the complexity. By further exploring the signal correlation in range direction, the complexity of the PCD algorithm can be reduced to a level similar to that of BPA. This work is out of the scope of this paper and will be reported separately.

\section{Performance Analysis and Simulation Results}

In this section, the GCW-SAR performance is evaluated in an airborne simulation scenario and compared with conventional FMCW-SAR, both using periodic chirp signals as the transmitted signals. Denote $N_{r}=T / T_{r}$ as the number of chirp periods over the aperture time $T$, where $T_{r}$ is the period of the chirp signal. The FMCW-SAR and the GCWSAR are assumed to operate in the same stripmap mode with the following airborne SAR parameters [6]: carrier frequency $10 \mathrm{GHz}$, speed of radar platform $70 \mathrm{~m} / \mathrm{s}$, platfrom altitude $7000 \mathrm{~m}$, antenna aperture $0.9 \mathrm{~m}$, and $R_{c}=8083 \mathrm{~m}$. Moreover, the $y$ and $x$ coordinates are normalized by the range and azimuth resolutions $\delta_{y}$ and $\delta_{x}$ respectively.

\section{A. Anti-Self-interference Performance in Digtal Domain}

Due to the FD operation in the GCW-SAR, the interference from the transmitted signal will impact on the imaging performance. Assuming that sufficient self-interference cancellation can be achieved by using existing joint propagation domain and analog-circuit domain cancellation techniques so that the reflected signal and self-interference are received within the ADC dynamic range. We only consider the residual selfinterference in digital domain in this subsection.

To analyze the anti-self-interference performance, the received signal with self-interference can be simply expressed as

$$
s_{s i}(t)=s_{r}(t)+\int_{0}^{\tau_{g}} g(\tau) \cdot s(t-\tau) e^{-j \frac{4 \pi}{\lambda} \tau c} d \tau
$$

where $g(t)$ and $\tau_{g}$ denote the interference channel impulse response and the maximum time delay of the self-interference respectively. When reconstructing the image for a point $\left(x_{m}, y_{n}\right)$ in the beam footprint, the output of the matched filter can be expressed as

$$
\begin{aligned}
& I_{s i}\left(x_{m}, y_{n}\right) \\
& =I\left(x_{m}, y_{n}\right)+\int_{0}^{\tau_{g}} g(\tau) \int_{\frac{x_{m}}{v}-\frac{T}{2}}^{\frac{x_{m}}{v}+\frac{T}{2}} s(t-\tau) \\
& s^{*}\left(t-\frac{2 r\left(t, x_{m}, y_{n}\right)}{c}\right) e^{j \frac{4 \pi}{\lambda}\left(r\left(t, x_{m}, y_{n}\right)-c \tau\right)} d t d \tau .
\end{aligned}
$$

The integral with regard to $t, \int_{\frac{x_{m}}{v}-\frac{T}{2}}^{\frac{x_{m}}{v}} s(t-\tau) s^{*}(t-$ $\left.\frac{2 r\left(t, x_{m}, y_{n}\right)}{c}\right) e^{j \frac{4 \pi}{\lambda}\left(r\left(t, x_{m}, y_{n}\right)-c \tau\right)} d t$, represents an ambiguity function of a hypothetical point scatterer with range $0<$ $\frac{1}{2} c \tau<\frac{1}{2} c \tau_{g}$. Since $\frac{1}{2} c \tau_{g}$ is much smaller than the slant range $r\left(t, x_{m}, y_{n}\right)$, only small sidelobes of the ambiguity function will be superimposed on $I\left(x_{m}, y_{n}\right)$.

In the first simulation experiment, the impact of the selfinterference on the GCW-SAR imaging performance is investigated. Firstly, we define the signal to interference power ratio as

$$
S I R=10 \cdot \log _{10}\left(\frac{P_{r}}{P_{i}}\right)
$$




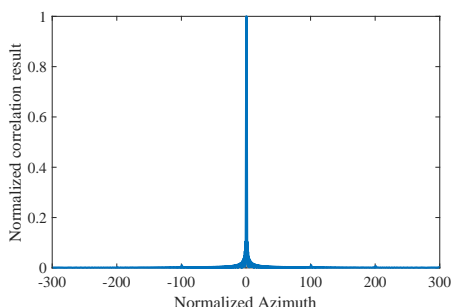

(a)

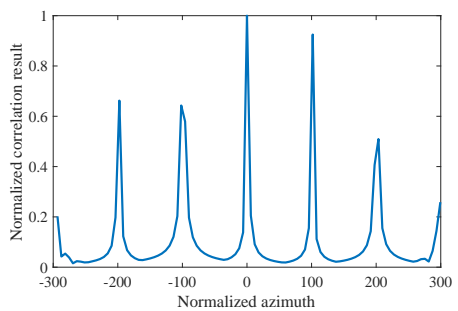

(d)

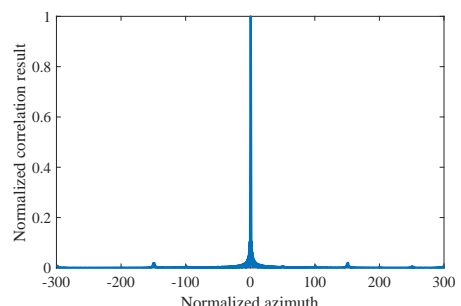

(b)

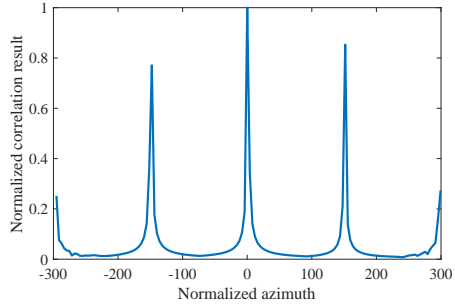

(e)

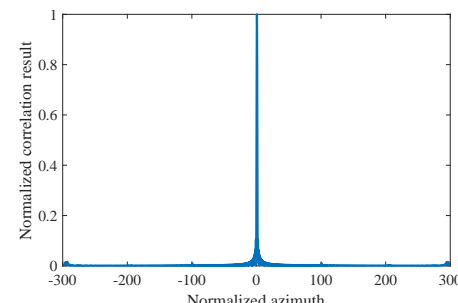

(c)

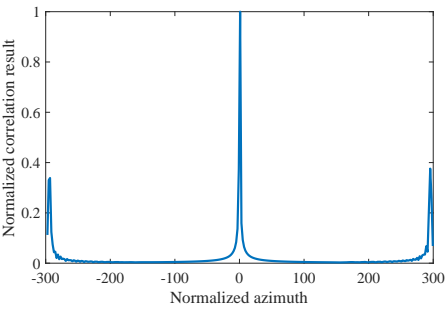

(f)

Fig. 5. Azimuth imaging comparison between GCW-SAR and FMCW-SAR with different PRFs: (a), (b), and (c) show the performance of GCW-SAR with $\mathrm{PCD}$ algorithm at $P R F=0.26 \mathrm{~Hz}, 39 \mathrm{~Hz}$, and $76.6 \mathrm{~Hz}$ respectively; (d), (e) and (f) show the performance of FMCW-SAR at $P R F=13 \mathrm{~Hz}, 39 \mathrm{~Hz}$, and $76.6 \mathrm{~Hz}$ respectively.

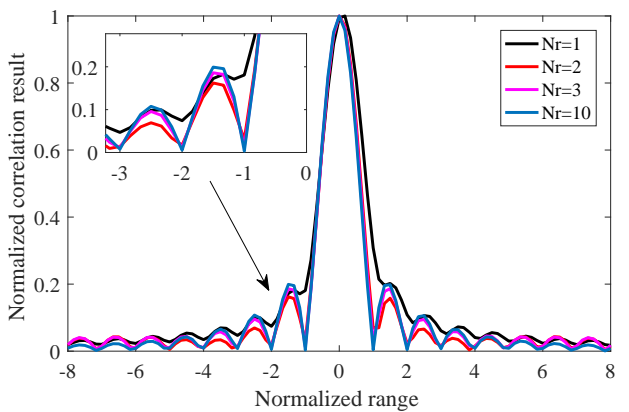

Fig. 6. Range resolution with different transmitted repetition interval.

where $P_{r}$ and $P_{i}$ are the average powers of the received echoes and the self-interference respectively. Assuming the self-interference directly comes from the transmitter and ignoring the white noise, Fig. 4 shows the imaging results for a point at $(0,0)$ with SIR $=-45 d B,-55 d B$ and $-65 d B$ respectively in an airborne platform. It is seen that the GCW-SAR is highly resistant to self-interference. With SIR higher than $-45 d B$, the self-interference is negligible. Due to the GCW-SAR's superb anti-self-interference ability, the SIC requirement in a practical system can be surely satisfied even the actual SIC level achieved in the analog-circuit domain is affected by some negative effects such as non-linearity and phase noise in the radar electronics.

\section{B. Impact of Pulse Repetition Period}

In the second simulation experiment, we investigate how the period of the chirp signal $T_{r}$ affects the imaging performance in GCW-SAR and FMCW-SAR respectively. With the radar parameters given at the beginning of this section, the length of the synthetic aperture is almost $600 \delta_{x}$, the lowest possible value of PRF is $0.26 \mathrm{~Hz}$ since in this case the period $T_{r}$ is equal to the aperture time $T$, and the unambiguous PRF for the FMCW-SAR is $78 \mathrm{~Hz}$.

The PRF, which is equal to $1 / T_{r}=N_{r} / T$, has little impact on azimuth resolution in GCW-SAR but significantly impacts on FMCW-SAR. Fig. 5 shows the imaging performance with different PRFs in GCW-SAR and FMCW-SAR systems for a point source located at $(0,0)$. It is clearly seen that the GCW-SAR performs much better than the FMCW-SAR which adopts the slow time sampling in azimuth. The change in PRF does not affect the azimuth resolution for the GCW-SAR, even in the lowest PRF case as shown in Fig. 5 (a). However, in FMCW-SAR, ambiguities will appear when the PRF is less than $78 \mathrm{~Hz}$. For instance, as seen in Fig. 5 (c) and 5 (f), the two ambiguities show up at $-295 \delta_{x}$ and $295 \delta_{x}$ symmetrically in FMCW-SAR image when the PRF is $76.6 \mathrm{~Hz}$, whereas they are hardly noticeable in the GCW-SAR image. Fig. 5 (d) and 5 (e) show the low PRF cases in FMCW-SAR where the PRFs are $13 \mathrm{~Hz}$ and $39 \mathrm{~Hz}$ respectively. It is apparent that ambiguities will appear periodically, thus leading to much deteriorated FMCW-SAR images. Therefore, GCW-SAR can break the limitation of minimum antenna area constraint and obtain a wide swath image with high azimuth resolution.

Low PRF may slightly degrade the mainlobe of the ambiguity function in range direction, but as shown in the following simulation, such degradation will be negligible after $N_{r}$ is larger than 3. With the same one-point imaging setup, Fig. 6 shows the image using PCD algorithm with $P=100$, but different $N_{r}$. We see that the range resolution is higher when $N_{r}$ is larger. When $N_{r}$ is larger than 3 , there is no significant improvement any more. 


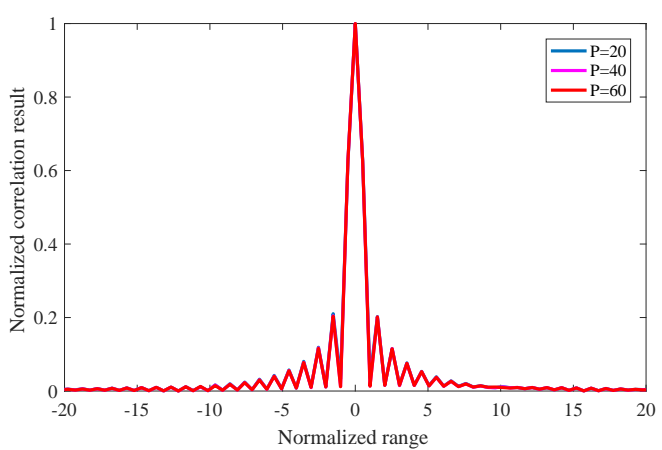

(a)

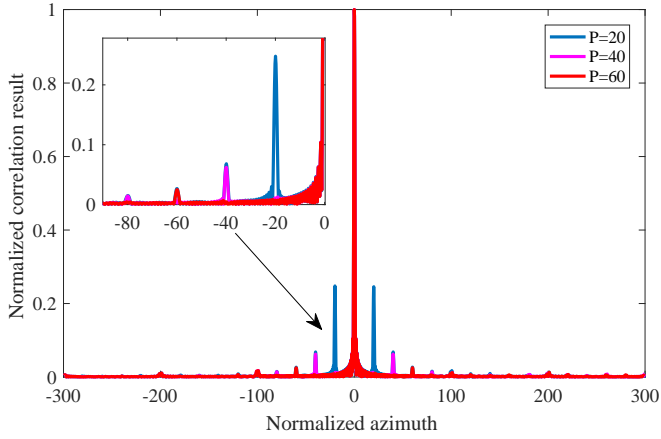

(b)

Fig. 7. Imaging performance with different number of segments $P$ : (a) image in range direction; (b) image in azimuth direction.

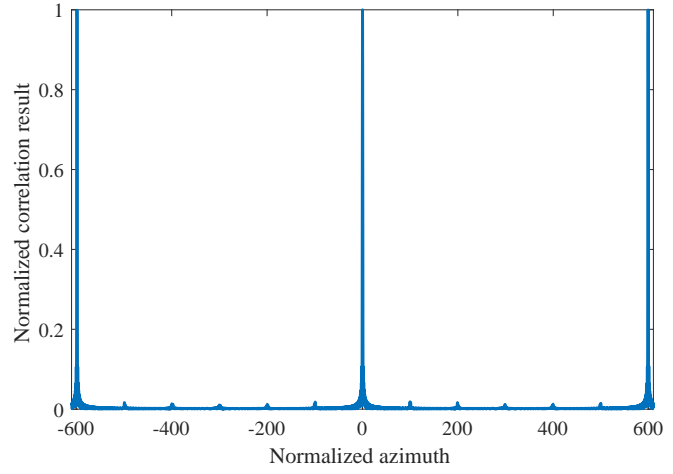

Fig. 8. A large image in azimuth.

\section{Impact of Segmention on PCD Algorithm}

The imaging performance of the PCD algorithm with different numbers of segments $P$ is also investigated. With $N_{r}$ set to 100 , the reconstructed images in the one-point imaging simulation are shown in Fig. 7 (a) and (b) in range and azimuth directions respectively. In range direction, Fig. 7 (a) shows that the PCD algorithm performs well regardless of $P$ since the linear piecewise approximation is only applied in azimuth direction.

The imaging performance in azimuth is closely associated with the number of segments $P$ since the range curve is approximated as $P$ linear segments. As seen from Fig. 7 (b), the azimuth ambiguities caused by linear approximation are alleviated by the increasing number of segmentation $P$ since the shorter segments approximate the range curve more accurately. On the other hand, the computational cost of PCD algorithm strongly depends on the $P$. It is also noticed that the unwanted spikes become weaker and can be neglected when $P \geq 60$, which is suitable for the implementation of a real system.

\section{Large Image Reconstruction in Azimuth}

To achieve a large image in azimuth, conventional SAR reconstructs images along the track block by block and then performs image stitching and registration techniques to form a large one [38]. In contrast, GCW-SAR image is reconstructed recursively and such a large image can be achieved directly, thus saving significant operations. For simplicity, we only consider imaging three targets in the azimuth direction and the three targets are located at $-600 \delta_{x}, 0$, and $600 \delta_{x}$ respectively. Both $P$ and $N_{r}$ are set to 100 . Fig. 8 shows the reconstructed GCW-SAR image where $x$-axis spans from $-610 \delta_{x}$ to $610 \delta_{x}$, almost twice as long as the synthetic aperture. It is evident that the three targets are well distinguished without ambiguities.

\section{E. Performance of PCD Algorithm}

In the final simulation experiment, we compare the imaging performance with the ideal matched-filtering based algorithm and the PCD algorithm in GCW-SAR, and investigate the ambiguities caused by linear approximation. The reconstructed images with point targets and extended targets are shown in Fig. 9 and Fig. 10 respectively. $N_{r}$ is set to 100 in the simulation.

Fig. 9 (a), 9 (b) and 9 (c) are the images of point targets with PCD algorithm when $P=10, P=20$ and $P=50$ respectively, and Fig. 9 (d) is the image of point targets with the ideal matched-filtering algorithm. It is clearly seen that the targets are correctly imaged based on the PCD algorithm when $P$ is increased to 50. Compared with the image using the matched-filtering shown in Fig. 9 (d), the only degradation of the image quality with the PCD algorithm appears in the azimuth direction when $P$ is small due to the linear approximation. In range direction, the targets can be distinguished clearly even when $P=10$. Such degradation can be neglected if a suitable number of segments $P$ is used.

The extended target whose size is large compared with a resolution cell can be also correctly imaged based on the PCD algorithm when $P$ is large enough. Fig. 10 (a), 10 (b) and 10 (c) show the imaging performance when $P=10, P=20$, and $P=50$ respectively, and Fig. 10 (d) is the image of extended target with the ideal matched-filter algorithm. Apparently, the PCD algorithm achieves the same performance for both point target and extended target and the linear approximation can only degrade the image quality in azimuth.

Therefore, the PCD algorithm is an effective method to reduce the computational complexity while maintaining superb image quality. 


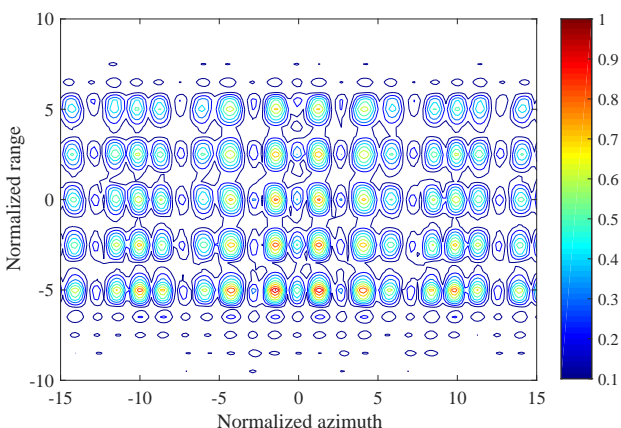

(a)

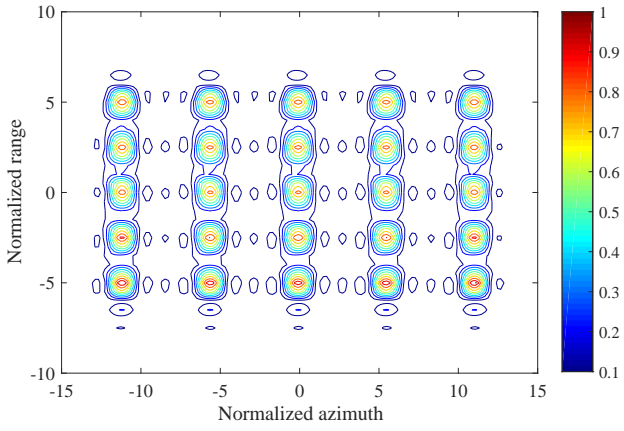

(c)

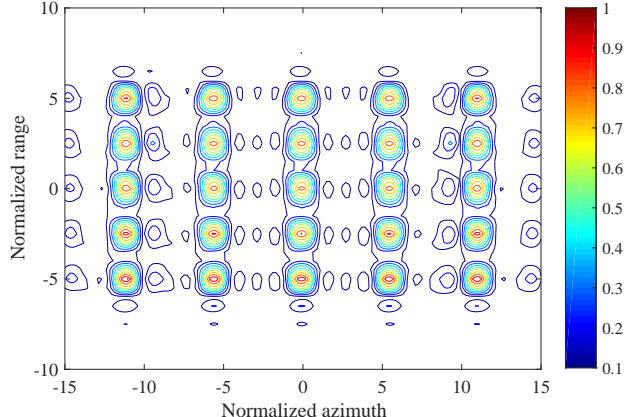

(b)

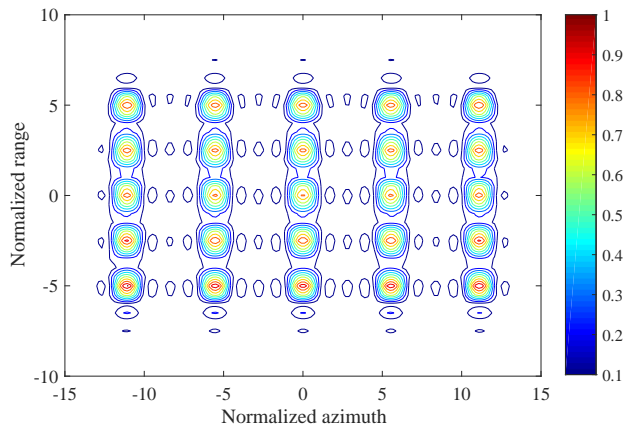

(d)

Fig. 9. Imaging quality comparision between the PCD algorithm and the BPA with multiple point targets in GCW-SAR: (a) image with PCD algorithm where $P=10$; (b) image with PCD algorithm where $P=20$; (c) image with PCD algorithm where $P=50$; (d) ideal matched-filtering based image.

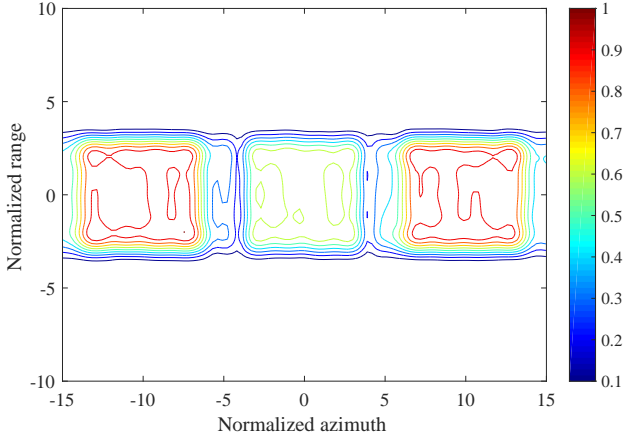

(a)

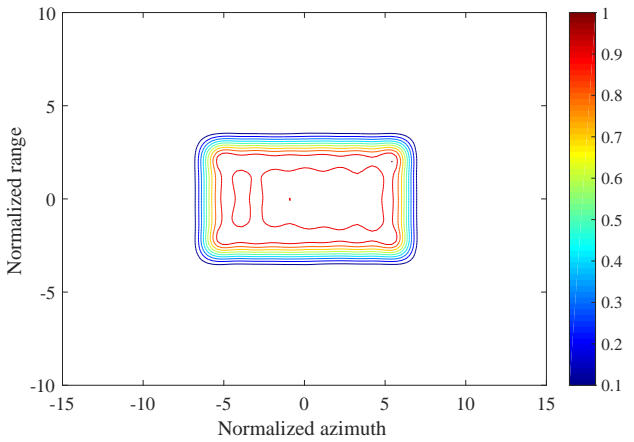

(c)

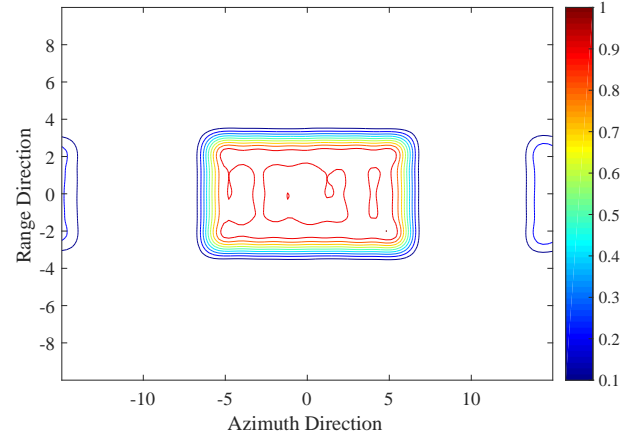

(b)

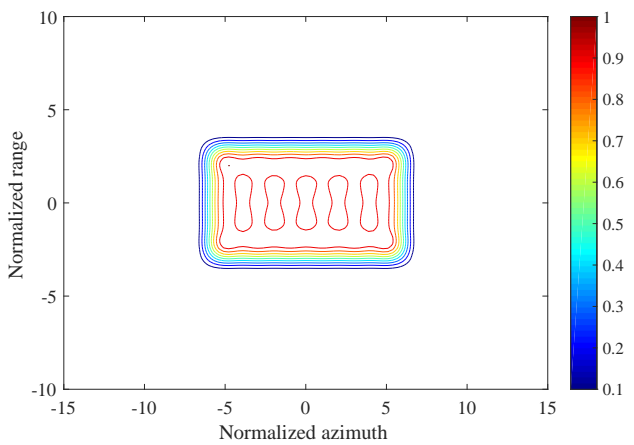

(d)

Fig. 10. Imaging quality comparision between the PCD algorithm and the BPA with an extended target in GCW-SAR: (a) image with PCD algorithm where $P=10$; (b) image with PCD algorithm where $P=20$; (c) image with PCD algorithm where $P=50$; (d) ideal matched-filtering based image. 


\section{CONCLUSIONS}

We have proposed a novel SAR concept with full duplex operation and continuous wave signalling. We have also proposed a fast imaging algorithm to reduce the computational complexity. In this GCW-SAR system, transmission and reception are conducted at the same time in the same frequency band by employing appropriate self-interference cancellation techniques and the continuously received signals can be extracted and stored as one-dimensional raw data. After correlating with location dependent reference signals, the one-dimensional raw data can be efficiently processed to reconstruct the image. The imaging performance is analyzed and simulated in terms of self-interference resistance in digital domain, impacts of transmitted signal designs and different parameters used in the different imaging algorithms. As shown from the analytical and simulation results, the proposed new SAR concept and PCD algorithm offer many advantages over conventional pulsed and CW SARs. Firstly, the radar receives more reflected signal energy with $\mathrm{CW}$ signalling, hence retaining the same advantages as FMCWSAR; Secondly, the sampling in azimuth will no longer be restricted, thus eliminating a number of inherent limitations in conventional systems; Thirdly, the system configuration and transmitted signal design are much more flexible. These advantages make the new SAR system a significant advance in SAR technology.

The work presented in this paper establishes a solid theoretical foundation for next generation imaging radars. The proposed GCW-SAR can be combined with the multipleinput and multiple-output radar to further improve the system performance, such as 3-D imaging suitable for the non-flat terrain. Based on the PCD algorithm, more new active and passive SAR configurations can be further designed to suit different performance requirements. In our future work, we will continue new SAR system designs and develop more practical algorithms for a wider range of applications.

\section{REFERENCES}

[1] J. H. Gonzalez, M. Bachmann, R. Scheiber, and G. Krieger, "Definition of ICESat Selection Criteria for Their Use as Height References for TanDEM-X," IEEE Transactions on Geoscience and Remote Sensing, vol. 48, no. 6, pp. 2750-2757, June 2010.

[2] X. M. Li, S. Lehner, and T. Bruns, "Ocean Wave Integral Parameter Measurements Using Envisat ASAR Wave Mode Data," IEEE Transactions on Geoscience and Remote Sensing, vol. 49, no. 1, pp. 155-174, Jan 2011.

[3] C. Clemente and J. J. Soraghan, "Vibrating Target Micro-Doppler Signature in Bistatic SAR With a Fixed Receiver," IEEE Transactions on Geoscience and Remote Sensing, vol. 50, no. 8, pp. 3219-3227, Aug 2012.

[4] A. Meta, P. Hoogeboom, and L. P. Ligthart, "Signal Processing for FMCW SAR,' IEEE Transactions on Geoscience and Remote Sensing, vol. 45, no. 11, pp. 3519-3532, Nov 2007.

[5] A. Currie and M. A. Brown, "Wide-swath SAR," IEE Proceedings F Radar and Signal Processing, vol. 139, no. 2, pp. 122-135, April 1992.

[6] A. Meta, "Signal processing of fmcw Synthetic Aperture Radar Data," 2006.

[7] J. Mittermayer, A. Moreira, and O. Loffeld, "Spotlight SAR Data Processing Using the Frequency Scaling Algorithm," IEEE Transactions on Geoscience and Remote Sensing, vol. 37, no. 5, pp. 2198-2214, Sep 1999.
[8] A. Moreira, J. Mittermayer, and R. Scheiber, "Extended Chirp Scaling Algorithm for Air- and Spaceborne SAR Data Processing in Stripmap and ScanSAR Imaging Modes," IEEE Transactions on Geoscience and Remote Sensing, vol. 34, no. 5, pp. 1123-1136, Sep 1996.

[9] J. H. Kim, M. Younis, A. Moreira, and W. Wiesbeck, "A Novel Ofdm Chirp Waveform Scheme for Use of Multiple Transmitters in SAR," IEEE Geoscience and Remote Sensing Letters, vol. 10, no. 3, pp. 568572, May 2013

[10] J. Wang, X. D. Liang, L. Y. Chen, and K. Li, "A Novel Space-Time Coding Scheme Used for MIMO-SAR Systems," IEEE Geoscience and Remote Sensing Letters, vol. 12, no. 7, pp. 1556-1560, July 2015.

[11] W. Q. Wang, "Space-Time Coding MIMO-OFDM SAR for HighResolution Imaging," IEEE Transactions on Geoscience and Remote Sensing, vol. 49, no. 8, pp. 3094-3104, Aug 2011.

[12] G. D. Callaghan and I. D. Longstaff, "Wide-swath Space-borne SAR Using A Quad-element Array," IEE Proceedings - Radar, Sonar and Navigation, vol. 146, no. 3, pp. 159-165, Jun 1999.

[13] G. Krieger, N. Gebert, and A. Moreira, "Multidimensional Waveform Encoding: A New Digital Beamforming Technique for Synthetic Aperture Radar Remote Sensing," IEEE Transactions on Geoscience and Remote Sensing, vol. 46, no. 1, pp. 31-46, Jan 2008.

[14] G. Krieger, "MIMO-SAR: Opportunities and Pitfalls," IEEE Transactions on Geoscience and Remote Sensing, vol. 52, no. 5, pp. 2628-2645, May 2014.

[15] N. Gebert, G. Krieger, and A. Moreira, "Digital Beamforming on Receive: Techniques and Optimization Strategies for High-Resolution Wide-Swath SAR Imaging," IEEE Transactions on Aerospace and Electronic Systems, vol. 45, no. 2, pp. 564-592, April 2009.

[16] D. Bharadia, E. McMilin, and S. Katti, "Full Duplex Radios," $A C M$ SIGCOMM Computer Communication Review, vol. 43, no. 4, pp. 375386, 2013.

[17] S. Hong, J. Brand, J. I. Choi, M. Jain, J. Mehlman, S. Katti, and P. Levis, "Applications of Self-Interference Cancellation in 5G and Beyond," IEEE Communications Magazine, vol. 52, no. 2, pp. 114-121, February 2014.

[18] A. Sabharwal, P. Schniter, D. Guo, D. W. Bliss, S. Rangarajan, and R. Wichman, "In-Band Full-Duplex Wireless: Challenges and Opportunities," IEEE Journal on Selected Areas in Communications, vol. 32, no. 9, pp. 1637-1652, Sept 2014

[19] J. I. Choi, M. Jain, K. Srinivasan, P. Levis, and S. Katti, "Achieving Single Channel, Full Duplex Wireless Communication," in Proceedings of the sixteenth annual international conference on Mobile computing and networking. ACM, 2010, pp. 1-12.

[20] M. A. Khojastepour, K. Sundaresan, S. Rangarajan, X. Zhang, and S. Barghi, "The Case for Antenna Cancellation for Scalable Full-Duplex Wireless Communications," in Proceedings of the 10th ACM Workshop on Hot Topics in Networks. ACM, 2011, p. 17.

[21] E. Everett, A. Sahai, and A. Sabharwal, "Passive Self-Interference Suppression for Full-Duplex Infrastructure Nodes," IEEE Transactions on Wireless Communications, vol. 13, no. 2, pp. 680-694, 2014.

[22] E. Everett, "Full-Duplex Infrastructure Nodes: Achieving Long Range with Half-Duplex Mobiles," Ph.D. dissertation, Rice University, 2012.

[23] X. Huang and Y. J. Guo, "Radio Frequency Self-Interference Cancellation With Analog Least Mean-Square Loop,' IEEE Transactions on Microwave Theory and Techniques, vol. PP, no. 99, pp. 1-15, 2017.

[24] J. Zhou, T. H. Chuang, T. Dinc, and H. Krishnaswamy, "Integrated Wideband Self-Interference Cancellation in the RF Domain for FDD and Full-Duplex Wireless," IEEE Journal of Solid-State Circuits, vol. 50, no. 12, pp. 3015-3031, Dec 2015.

[25] M. P. Chang, C. L. Lee, B. Wu, and P. R. Prucnal, "Adaptive Optical Self-Interference Cancellation Using a Semiconductor Optical Amplifier," IEEE Photonics Technology Letters, vol. 27, no. 9, pp. 1018-1021, May 2015.

[26] A. F. Yegulalp, "Fast Backprojection Algorithm for Synthetic Aperture Radar," in Proceedings of the 1999 IEEE Radar Conference. Radar into the Next Millennium (Cat. No.99CH36249), 1999, pp. 60-65.

[27] S. Xiao, D. C. Munson, S. Basu, and Y. Bresler, "An N2logN BackProjection Algorithm for SAR Image Formation," in Conference Record of the Thirty-Fourth Asilomar Conference on Signals, Systems and Computers (Cat. No.00CH37154), vol. 1, Oct 2000, pp. 3-7 vol.1.

[28] L. M. H. Ulander, H. Hellsten, and G. Stenstrom, "Synthetic-Aperture Radar Processing Using Fast Factorized Back-Projection," IEEE Transactions on Aerospace and Electronic Systems, vol. 39, no. 3, pp. 760776, July 2003

[29] Y. F. Shao, R. Wang, Y. K. Deng, Y. Liu, R. Chen, G. Liu, and O. Loffeld, "Fast Backprojection Algorithm for Bistatic SAR Imaging," 
IEEE Geoscience and Remote Sensing Letters, vol. 10, no. 5, pp. 10801084, Sept 2013.

[30] V. T. Vu and M. I. Pettersson, "Fast Backprojection Algorithms Based on Subapertures and Local Polar Coordinates for General Bistatic Airborne SAR Systems," IEEE Transactions on Geoscience and Remote Sensing, vol. 54, no. 5, pp. 2706-2712, May 2016.

[31] A. Ribalta, "Time-Domain Reconstruction Algorithms for FMCWSAR," IEEE Geoscience and Remote Sensing Letters, vol. 8, no. 3, pp. 396-400, May 2011.

[32] Z. Li, J. Wang, and Q. H. Liu, "Frequency-Domain Backprojection Algorithm for Synthetic Aperture Radar Imaging," IEEE Geoscience and Remote Sensing Letters, vol. 12, no. 4, pp. 905-909, April 2015.

[33] Z. Jiang, F. Huang, J. Wan, and Z. Cheng, "Modified Frequency Scaling Algorithm for FMCW SAR Data Processing," Chinese Journal of Aeronautics, vol. 20, no. 4, pp. 339-345, 2007.

[34] I. S. Merrill, Radar Handbook. NewYork: McGraw Hill, 1990, ch. 21.4.

[35] W. Zhou, H. Chen, W. Jiang, and H. Wang, "Generalized Ambiguity Function Analysis of MIMO SAR," in 2012 IEEE 11th International Conference on Signal Processing, vol. 3, Oct 2012, pp. 1724-1728.

[36] D. Tarchi, K. Lukin, J. Fortuny-Guasch, A. Mogyla, P. Vyplavin, and A. Sieber, "SAR Imaging with Noise Radar," IEEE Transactions on Aerospace and Electronic Systems, vol. 46, no. 3, pp. 1214-1225, July 2010.

[37] Y. Na, Y. Lu, and H. Sun, "A Comparison of Back-Projection and Range Migration Algorithms for Ultra-Wideband SAR Imaging," in Fourth IEEE Workshop on Sensor Array and Multichannel Processing, 2006., July 2006, pp. 320-324.

[38] N. Li, R. Wang, Y. Deng, T. Zhao, W. Wang, and H. Zhang, "Processing Sliding Mosaic Mode Data With Modified Full-Aperture Imaging Algorithm Integrating Scalloping Correction," IEEE Journal of Selected Topics in Applied Earth Observations and Remote Sensing, vol. 10 no. 5, pp. 1804-1812, May 2017. 